\title{
High-Frequency Impedance of Driven Superlattices
}

\author{
A.-K. Jappsen ${ }^{a}$, A. Amann ${ }^{a}$, A. Wacker ${ }^{a}$, E. Schomburg ${ }^{b}$, E. Schöll ${ }^{a}$ \\ ${ }^{a}$ Institute for Theoretical Physics, Technical University of Berlin, Hardenbergstr. 36, D-10623 Berlin, Germany \\ ${ }^{b}$ Institute for Applied Physics, Universität Regensburg, D-93040 Regensburg, Germany
}

(November 10, 2018)

\begin{abstract}
The complex impedance of a semiconductor superlattice biased into the regime of negative differential conductivity and driven by an additional $\mathrm{GHz}$ ac voltage is computed. From a simulation of the nonlinear spatio-temporal dynamics of traveling field domains we obtain strong variations of the amplitude and phase of the impedance with increasing driving frequency. These serve as fingerprints of the underlying quasiperiodic or frequency locking behavior. An anomalous phase shift appears as a result of phase synchronization of the traveling domains. If the imaginary part of the impedance is compensated by an external inductor, both the frequency and the intensity of the oscillations strongly increase.
\end{abstract}

72.20.Ht,73.61.-r

Semiconductor superlattices (SL) show pronounced negative differential conductivity (NDC). If the total bias is chosen such that the average electric field is in the NDC region, stable inhomogeneous field distributions (field domains) 1 or self-sustained oscillations 3 with frequencies up to $150 \mathrm{GHz}$ at room temperature $\mathrm{\theta}$ appear. Which of these scenarios occurs depends on bias, doping, temperature, and the properties of the injecting contact5 10. For a recent overview see Ref.11.

In order to apply the self-sustained oscillations as a high-frequency generator in an electronic device, it is crucial to know the response of the SL in an external circuit. A key ingredient for the analysis is the complex impedance of the SL in the respective frequency range. This is the subject of this paper where the complex impedance is evaluated numerically by imposing an additional ac bias to the SL. The interplay of the selfsustained oscillations and the external frequency causes a variety of interesting phenomena such as frequency locking, quasi-periodic and chaotic behavior which has been extensively studied both theoretically 124 and experimentally 15,16 . In contrast to those studies we focus on the response to the circuit and concurrent phase synchronization phenomena in this work.

We describe the dynamical evolution of the SL by rate equations for the electron densities in the quantum wells, together with Poisson's equation for the electric fields. The current densities $J_{j \rightarrow j+1}$ between adjacent quantum wells are evaluated within the model of sequential tunneling, and Ohmic boundary conditions with a contact conductivity $\sigma$ are used. For details see Refs.1011. Here we apply a periodic bias signal $U(t)=U_{\mathrm{dc}}+U_{\mathrm{ac}} \sin \left(2 \pi \nu_{1} t\right)$ and study the total current $I(t)=A \sum_{j=0}^{N} J_{j \rightarrow j+1} /(N+1)$. In particular we consider the Fourier component $I_{\mathrm{ac}}\left(\nu_{1}\right) \sin \left(2 \pi \nu_{1} t-\phi\right)$ which gives the complex impedance

$$
Z\left(\nu_{1}\right)=\frac{U_{\mathrm{ac}}}{I_{\mathrm{ac}}\left(\nu_{1}\right)} e^{i \phi} .
$$

As an example we consider the SL structure studied in
Ref.6. It consists of $N=120 \mathrm{GaAs}$ wells of width 4.9 $\mathrm{nm}$ separated by $1.3 \mathrm{~nm}$ AlAs barriers. The sample is n-doped with a density of $8.7 \times 10^{10} \mathrm{~cm}^{-2}$ per period and the sample cross section is $A=64(\mu \mathrm{m})^{2}$. We estimate the energy broadening $\Gamma=15 \mathrm{meV}$, which effectively gives the sum of phonon, impurity and interface roughness scattering rates. All calculations are performed at room temperature.

In Fig. 11a we display the calculated current-voltage characteristic for $U_{\mathrm{ac}}=0$. While for large values of the contact conductivity $\sigma$ stationary field domains occur, self-sustained current oscillations (where pairs of accumulation and depletion fronts travel through the sample) are found for lower $\sigma$ (Fig. 1 b). This scenario is quite general for moderately doped samplest 11 . In the following we use $\sigma=20 \mathrm{~A} / \mathrm{Vm}$ giving best agreement with the measured current-voltage characteristic.

At $U_{\mathrm{dc}}=2 \mathrm{~V}$, the self-sustained oscillations exhibit a frequency of $\nu_{0}=2.1 \mathrm{GHz}$. Now we study the change in the current signal by imposing an additional ac-bias amplitude $U_{\text {ac }}$ with frequency $\nu_{1}$. Fig. 2 displays the absolute value and the phase of the complex impedance given by Eq. (1). For both strengths of the ac amplitude one observes strong variations in the amplitude and phase of $Z$. In particular one observes pronounced minima in $|Z|$ and a corresponding monotonic increase in $\phi$ around frequencies $\nu_{1}$ which are approximately integer multiples of $\nu_{0}$. This is due to frequency locking 17 , where the external frequency $\nu_{1}$ modifies the main oscillation frequency to $\nu_{0}^{*}$ such that $\nu_{1} / \nu_{0}^{*}$ is a rational number. While locking occurs in a rather wide frequency range for $\nu_{1} / \nu_{0}^{*} \in \mathbb{N}$, locking into rational numbers with larger denominators are less easy to observe (The corresponding widths of the locking intervals roughly follow the position in the Farey-tree of the rational numbers, see 17 . The width of the locking ranges also increases with $U_{\mathrm{ac}}$.) A more detailed examination indicates that the local minima in $|Z|$ correspond to the locking regions, and $\phi$ increases with $\nu_{1}$ in those intervals, while a decrease is frequently ob- 
served outside these ranges where quasi-periodic or possibly chaotic beharipr occurs. Thus the presence of several locking intervals 16 explains the variety of peaks.

For high frequencies $\nu_{1} \gg \nu_{0}$ the variations of $Z$ become less pronounced and $|Z| \sim 300 \Omega$ and $\phi \approx-\pi / 2$ is observed. This corresponds to a capacitive current with an effective frequency-dependent capacitance $C_{\text {eff }}\left(\nu_{1}\right) \approx$ $1 /\left(2 \pi \nu_{1}|Z|\right)$. At $\nu_{1}=10 \mathrm{GHz}$ this gives $C_{\text {eff }} \approx 50 \mathrm{fF}$. This capacitance results from the interaction with the internal front dynamics in the SL structure; the intrinsic sample capacitance $\epsilon_{r} \epsilon_{0} A /(N d)=10 \mathrm{fF}$ is significantly smaller.

While we have only shown results for $U_{\mathrm{dc}}=2 \mathrm{~V}$ and $\sigma=20 \mathrm{~A} / \mathrm{Vm}$ here, we checked that the features discussed above neither change for different biases nor different contact conductivities, albeit the main oscillation frequency $\nu_{0}$ and the locking ranges change slightly.

Now we focus on the $1 / 1$ locking region which is of interest if the device is used as an oscillator. In Fig. 3 we show the current and bias signal for different frequencies $\nu_{1}$. In parts (a) $\left(\nu_{1}=1.95 \mathrm{GHz}\right)$ and (f) $\left(\nu_{1}=2.3\right.$ $\mathrm{GHz}$ ) no complete locking occurs and the current does not exhibit a periodic signal. Between these frequencies the current signal is periodic with a frequency $\nu_{0}^{*}=\nu_{1}$ imposed by the external bias. While the current peaks occur around the minima of the external bias for $\nu_{1}=2$ $\mathrm{GHz}$ (corresponding to $\phi \approx-\pi$ ), the delay between the current peaks and the bias maxima decreases with frequency until they are approximately in phase at the upper boundary of the locking range at $\nu_{1}=2.25 \mathrm{GHz}$ (corresponding to $\phi \approx 0$ ). It is intriguing to note that this behavior is opposite to the response of a damped linear oscillator, where the phase between the driving signal and the response shifts from 0 to $\pi$ with increasing driving frequency. The current peaks correspond to the formation of a domain at the emitter, while during the current minima the domain traverses the sample. The domain transit velocity is larger for smaller voltage. The phase shift between current and voltage adjusts such that the domain velocity increases with increasing driving frequency during the whole locking interval. This is a phase synchronization effect induced by the domain dynamics.

Let us analyze the behavior close to the onset of the locking interval in detail, see Fig. A. The main frequency component of the current signal is given by $\nu_{0}^{*}$. In the locking region it is equal to the frequency of the driving bias $\nu_{1}$ such that $\nu_{0}^{*}-\nu_{1}$ vanishes in a finite range of $\nu_{1}$. In contrast, far away from the locking $\nu_{0}^{*}$ is essentially given by the free oscillation frequency $\nu_{0}$, thus $\nu_{0}^{*}-\nu_{1}$ exhibits a linear relation. Close to the boundaries of the locking region a square root behavior $\nu_{0}^{*}-\nu_{1} \propto \sqrt{\left|\nu_{1}-\nu_{1}^{\text {crit }}\right|}$ can be detected, which is a general feature of frequency locking, see, e.g. 18 .

Fig. tb shows the amplitude and the monotonically increasing phase of the impedance over the whole locking interval. We extract an impedance $Z \approx-i 150 \Omega$ for $\nu_{0}=2.1 G H z$. Now we compensate this impedance by an external circuit with an inductor of $L=10 \mathrm{nH}$ and a resistor of $25 \Omega$ in series with the SL. (The resistor may result both from connecting wires or due to radiation damping in a real device.) Fig. 5 shows that the oscillation mode is strongly affected: The frequency is increased to $\nu=5.6 \mathrm{GHz}$, the oscillation is more sinusoidal, and the current amplitude increases. Thus the device performance of the SL is strongly improved. This effect is due to a different oscillation mode, where the domains are quenched such that they only traverse a small part of the SL. As the domain velocity is almost constant, the frequency increases.

We have shown that the amplitude and the phase of the complex impedance $Z$ exhibit strong variations with the driving frequency $\nu_{1}$. The distinct minima of the amplitude of the impedance correspond directly to the frequency locking intervals. In these regions the frequency components of the current at $\nu_{1}$ are particularly large, leading to small $|Z|$. The phase of the impedance is monotonically increasing with frequency from $-\pi$ to zero in the locking intervals, which constitutes a phase shift of $\pi$ compared to the standard behavior of a driven oscillator. These effects are caused by phase synchronization of the spatio-temporal dynamics of the traveling field domains in the NDC regime. Compensating the imaginary part of the impedance in an external circuit strongly improves the device performance of the SL.

Partial support from Sfb 555 is acknowledged.

${ }^{1}$ L. Esaki and R. Tsu, IBM J. Res. Develop. 14, 61 (1970).

${ }^{2}$ L. Esaki and L. L. Chang, Phys. Rev. Lett. 33, 495 (1974).

${ }^{3}$ J. Kastrup, R. Klann, H. T. Grahn, K. Ploog, L. L. Bonilla, J. Galán, M. Kindelan, M. Moscoso, and R. Merlin, Phys. Rev. B 52, 13761 (1995).

${ }^{4}$ E. Schomburg, R. Scheurer, S. Brandl, K. F. Renk, D. G. Pavel'ev, Y. Koschurinov, V. Ustinov, A. Zhukov, A. Kovsh, and P. S. Kop'ev, Electronics Letters 35, 1491 (1999).

5 A. Wacker, M. Moscoso, M. Kindelan, and L. L. Bonilla, Phys. Rev. B 55, 2466 (1997).

${ }^{6}$ M. Patra, G. Schwarz, and E. Schöll, Phys. Rev. B 57, 1824 (1998).

${ }^{7}$ D. Sánchez, M. Moscoso, L. L. Bonilla, G. Platero, and R. Aguado, Phys. Rev. B 60, 4489 (1999).

${ }^{8}$ J. Wang, B. Q. Sun, X. Wang, Y. Wang, W. Ge, and H. Wang, Appl. Phys. Lett. 75, 2620 (1999).

${ }^{9}$ H. Steuer, A. Wacker, E. Schöll, M. Ellmauer, E. Schomburg, and K. F. Renk, Appl. Phys. Lett. 76, 2059 (2000).

10 A. Amann, A. Wacker, L. L. Bonilla, and E. Schöll, Phys. Rev. E 63, 066207 (2001).

${ }^{11}$ A. Wacker, Phys. Rep. 357, 1 (2002).

12 O. M. Bulashenko and L. L. Bonilla, Phys. Rev. B 52, 7849 (1995).

13 J. C. Cao and X. L. Lei, Phys. Rev. B 60, 1871 (1999). 
${ }^{14}$ D. Sánchez, G. Platero, and L. L. Bonilla, Phys. Rev. B 63, 201306 (2001).

${ }^{15}$ Y. Zhang, J. Kastrup, R. Klann, K. Ploog, and H. T. Grahn, Phys. Rev. Lett. 77, 3001 (1996).

${ }^{16}$ E. Schomburg, K. Hofbeck, R. Scheurer, M. Haeussler, K. F. Renk, A.-K. Jappsen, A. Amann, A. Wacker, E. Schöll, D. G. Pavel'ev, and Y. Koschurinov, Phys. Rev. B submitted (2002).

${ }^{17}$ H. G. Schuster, Deterministic Chaos, 2 ed. (VCH Verlagsgesellschaft, Weinheim, 1988).

18 J. Peinke, J. Parisi, O. Rössler, and R. Stoop, Encounter with Chaos (Springer, Berlin, Heidelberg, 1992).
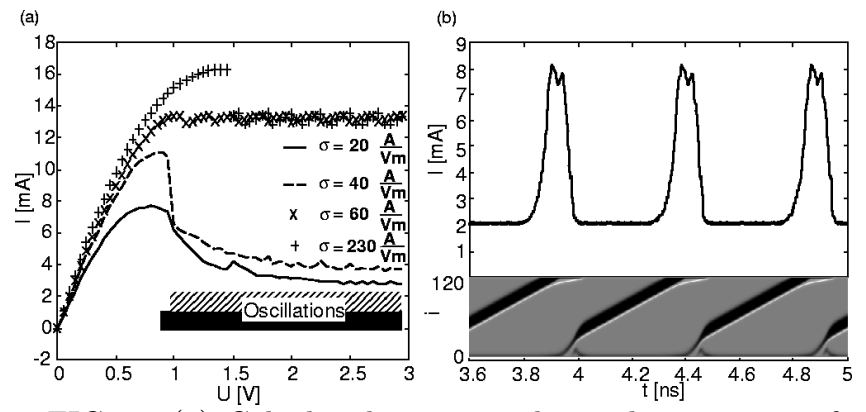

FIG. 1. (a) Calculated current-voltage characteristics for different contact conductivities $\sigma$, and $U_{\text {ac }}=0$. For $\sigma=20$ $\mathrm{A} / \mathrm{Vm}$ and $40 \mathrm{~A} / \mathrm{Vm}$ self-sustained current oscillations are found in the indicated bias ranges. In this case we display the time-averaged current. (b) Self-sustained oscillations for $U_{\mathrm{dc}}=2 \mathrm{~V}, \sigma=20 \mathrm{~A} / \mathrm{Vm}$. The time series of the current $I(t)$ and the space-time plot of the electron densities $n_{i}(t)$ in the quantum wells is shown. Black indicates low electron density (depletion front), white indicates high electron density (accumulation front). The emitter is at the bottom, the collector at the top.

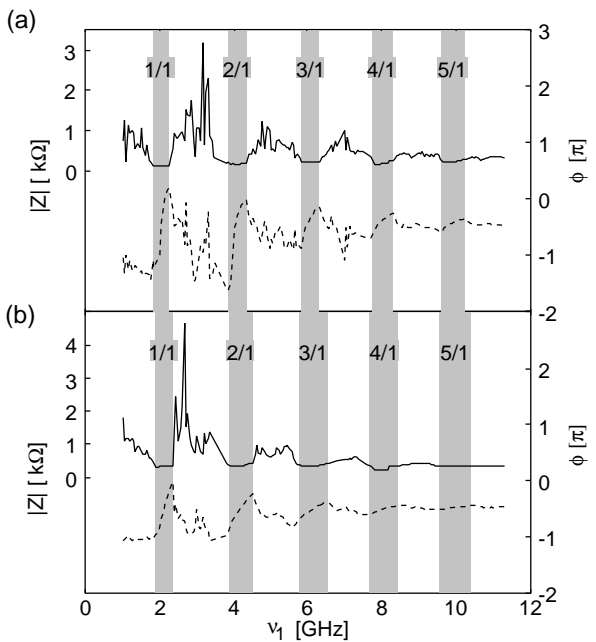

FIG. 2. Amplitude $|Z|$ (full line) and phase $\phi$ (dashed line) of the complex impedance $Z$ as a function of the driving frequency for two different values of the ac bias: (a) $U_{a c}=0.2 \mathrm{~V}$, (b) $U_{a c}=0.6 \mathrm{~V}$. The shaded areas indicate locking intervals marked by $\nu_{1} / \nu_{0}^{*}\left(U_{d c}=2 \mathrm{~V}, \sigma=20 \mathrm{~A} / \mathrm{Vm}\right)$.

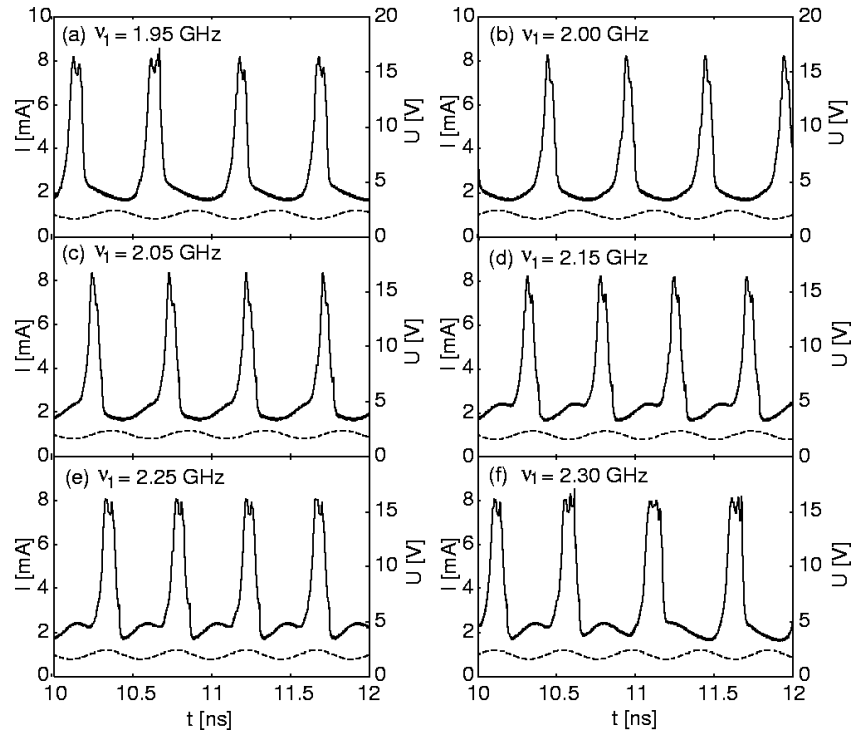

FIG. 3. Current $I$ (full line) and driving bias $U$ (dashed line) versus time for different driving frequencies $\nu_{1}$ $\left(U_{\mathrm{ac}}=0.4 \mathrm{~V}, U_{d c}=2 \mathrm{~V}\right)$.
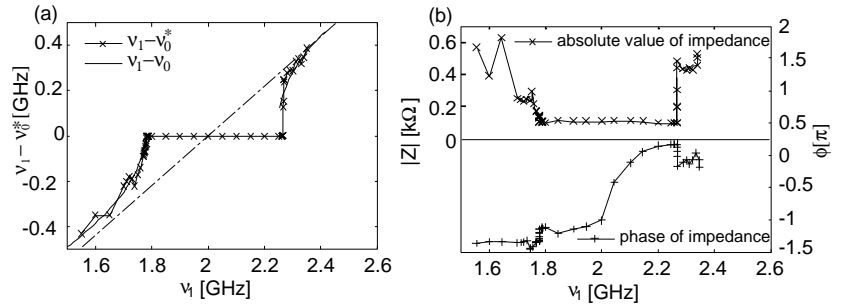

FIG. 4. (a) Shift of the fundamental frequency $\nu_{0}^{*}$ of the current signal and (b) amplitude and phase of the complex impedance as a function of the driving frequency $\nu_{1}$ in the vicinity of the $1 / 1$ locking region $\left(U_{\mathrm{ac}}=0.2 \mathrm{~V}, U_{d c}=2 \mathrm{~V}\right)$.

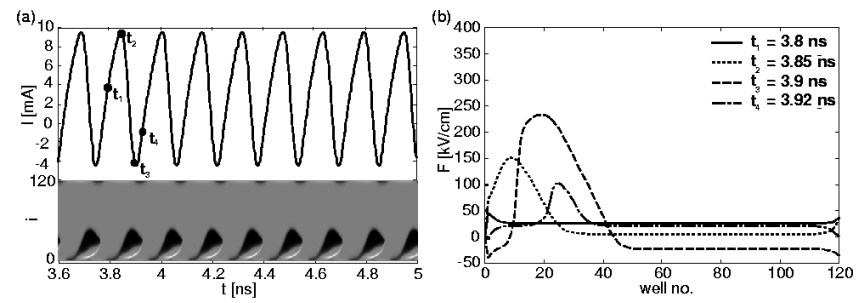

FIG. 5. Operation of the SL device with an inductor of 10 $\mathrm{nH}$ and a resistor of $25 \Omega$ in series $\left(U_{d c}=2 \mathrm{~V}, U_{\mathrm{ac}}=0\right)(\mathrm{a})$ Current versus time and space-time plot of the electron density in the SL (as in Fig.1b). (b) Electric field versus position for different times (as indicated in a). 